\title{
Leveraging object-orientation for real-time imaging systems
}

\author{
Colin J. Neill* \\ The Pennsylvania State University, Malvern, PA 19355, USA
}

\begin{abstract}
Imaging systems are traditionally developed using structured analysis and design techniques at best. Such approaches tend to be rigid with respect to changing needs, technologies, devices and algorithms - for example, when additional compression algorithms are needed or attached devices are changed large parts of software applications employing those techniques and interfacing with those devices must be modified to accommodate the change. In a larger perspective, these systems are difficult or impossible to reuse; each new problem requires a new solution.

This is generally undesirable and often not necessary, but only if best practices in software engineering are employed. These best practices have been explored and documented in detail with regard to object-oriented systems, which suggests that it is an appropriate paradigm to employ in the development of future imaging systems. This work examines these best practices, in the form of patterns and design principles, with reference to imaging systems and within the context of the Java imaging APIs.
\end{abstract}

(C) 2003 Elsevier Ltd. All rights reserved.

\section{Introduction and previous work}

The application of software engineering best practices in the development of real-time imaging systems appears to be an area in need of attention. The wider software engineering community is finally showing signs of maturity, as evidenced by improvements reported in the yearly Chaos Reports [1] but within that broad community there are still distinct pockets of engineers developing software systems with scant knowledge of, or adherence to, best practices. As Pereira points out "until recently the development of real-time systems was characterized as an 'occult art', where 'ad hoc' fashions and 'programming from scratch' techniques abounded" [2]. This is particularly problematic in application domains that experience continued advancement and evolution, such as real-time imaging. Developers of such systems are forced to redesign and re-write essentially the same system, but with slight modifications for the new application, algorithms or technologies employed. Function libraries exist that provide a plethora of image manipulation and transformation techniques for use here, but the underlying structure of each application often changes so little reuse is possible outside of these functions.

A more desirable alternative would be to tailor a general solution for each specific case, reusing as much

\footnotetext{
*Tel.: + 1-610-725-5285.

E-mail address: cjn6@psu.edu (C.J. Neill).
}

of the general case as possible and only redefining or adding the aspects that change. So, for example, if a new compression technique is required of a system, only the compression aspects of the general system are modified or extended and the rest of the application is unchanged. A development technique well-suited to this scenario is object-oriented analysis and design. Here the system is described as a set of classes that can be instantiated and work in cooperation with one another to fulfill the responsibilities of the application. While this is simply an alternate viewpoint of a software system, it allows the application of sophisticated principles and patterns that yield robust, maintainable, extensible and therefore reusable systems. In [3] the basic application of objectorientation $(\mathrm{OO})$ to imaging systems was investigated and compared against the more traditionally adopted practice (at least in the imaging field [4]) of structured analysis, structured design. This paper extends that work by examining the processes and practices that can be employed within the auspices of object-orientation in the building of reusable frameworks for imaging systems.

\section{OO and real-time}

Before the benefits of object-oriented approaches can be examined, some long-discussed issues regarding the suitability of object-oriented implementations for 
real-time systems must be examined. The most obvious is the inefficiencies of $\mathrm{OO}$ languages. This is really a comment aimed at the interpreted nature of Java. There are really two issues here, however. First, real-time does not, and should not be thought of, as real-fast. Realtime applications may indeed have tight time constraints to meet, but most of all, real-time systems should be deterministic (and we will return to this issue shortly). Second, Java is only one of several OO languages. In situations where performance is key $\mathrm{C}++$ may be more appropriate since it does not have Java's in-built garbage collection or interpretation. Another alternative is to utilize the real-time specification for Java [5].

Some more profound exceptions to OO were debated in a special section of Real-Time Systems journal [6] and included the "inheritance anomaly" and non-determinisms introduced by polymorphism and garbage collection.

\subsection{Inheritance anomaly}

The inheritance anomaly is the situation that arises when the introduction of a new method or the overriding of an inherited method in a subclass requires modification or redefinition of inherited properties. Synchronization inheritance anomalies are an extension of this concept where new constraints are imposed in inherited classes [7]. These anomalies are seen as problematic because they hinder the reuse of welldefined and verified real-time programs. There is a problem with this thinking, however. The assumption that inheriting features from a previously tested and trusted class obviates the need to test the new subclass is entirely flawed; each new context for an object offers new ways to misuse the object [8]. Beyond this naïve misunderstanding there is a more fundamental error, one common when inheritance is used as a convenient code reuse mechanism. Instances of derived classes should be substitutable for instances of their base classes [9], so must therefore be subtypes of the base class. Since a subtype cannot strengthen the pre-conditions of its supertype [10] the inheritance anomalies only occur when this central precept of $\mathrm{OO}$ is ignored.

Examining the example used by Bergmans and Aksit [7] it is clear where their problems arise. They describe how a Crane class that provides the basic functionality of a crane system has the additional abilities of storage and retrieval through its inheritance relationship with a BoundedBuffer class - an already questionable decision, since the basic heuristic for inheritance is whether the "is a" relationship holds and clearly the statement "a Crane is a BoundedBuffer" is not correct. They then discover that the BoundedBuffer behaviors must be constrained for real-time performance when used in the Crane, thus leading to modification of the inherited buffer features and the reported synchronization inheritance anomalies. However, these additional constraints violate the substitutability of Crane and BoundedBuffer (Crane is no longer a subtype of BoundedBuffer) so it is not surprising that anomalies occur.

\subsection{Non-determinism}

Perhaps the most substantive concerns regarding the use of $\mathrm{OO}$ for real-time are the non-deterministic aspects of the implementations. Java utilizes a garbage collector for memory management and reclamation that can preempt any thread at any time. This clearly introduces a level of non-determinism that would hinder usefulness. However, the real-time specification for Java [5] introduces a memory model that allows objects to be allocated outside of the heap in a memory area that is not subject to the garbage collector. Furthermore, alternative $\mathrm{OO}$ languages exist, such as $\mathrm{C}++$, that do not have an in-built garbage collector. In both cases memory management is left to the developer.

Of course there is still the issue of polymorphism and late binding, where method names are resolved at runtime. As will be evident in the later discussion, polymorphism is central to design for reuse so while we can avoid the late binding in some OO languages (class methods in $\mathrm{C}++$ are bound at compile time, for example) that would somewhat defeat the purpose. This does not necessarily present a stumbling block, however. Polymorphism is used as a mechanism to avoid repeated conditional logic, to support future extension without modification, and to allow interfaces and their implementations to vary independently. In all of these cases even though the implementation is not known at compile time, the interface is and therefore the object type. Returning to the above discussion concerning substitutability of subtypes, we see that proper subtypes must conform to the supertype not only in signature, but in constraints, thus each polymorphic choice must adhere to the constraints imposed on the base class, including any specified temporal constraints. This is not enforced automatically, the burden is on the developer to ensure conformance, but this is also true for each branch in a switch statement in procedural languages.

\section{Design for reuse}

We see then, that the object-oriented paradigm is compatible with real-time requirements and can progress with the consideration of leveraging the properties of OO for building reusable systems. The key to reuse is the identification of the mutable and immutable services or responsibilities of a system and isolating them. This is typically achieved by building frameworks.

A framework can be loosely defined as a set of interfaces (or abstract classes) and concrete classes that 
provide a stable set of core services to an application and can be extended for domain specific implementations through explicit extension mechanisms that isolate the variant aspects. Successful frameworks embody sincere distillations of core principles that underpin the object-oriented paradigm.

\subsection{OO principles}

When considering the benefits of object-oriented approaches to image processing applications it is easy to focus on the ideas of combining data and behavior into an encapsulated entity that better approximates the "things" in the problem domain and consider this closeness between reality and the modeling domain to be the central benefit of using objects. While this can be considered an advantage, the purported intuitiveness of the approach is, in fact, something of a fortuitous side effect. The real advantages of applying object-oriented paradigms are the future extensibility and reuse that can be attained, and the relative ease of future changes.

Several studies indicate that software systems are subject to near-continuous change: requirements change, merge, emerge, and mutate; target languages, platforms and architectures change; and most significantly the way the software is employed in practice changes [11]. This changeability places considerable burden on the software design. How can systems that must support such widespread change be built without compromising quality?

While object-oriented systems can be designed to be as rigid and resistant to extension and modification as in any other paradigm, OO has the ability to include distinct design elements that can cater to future changes and extensions. These "design patterns" were first introduced to the mainstream of software engineering practice by Gamma et al. [14]. The "Gang of Four (GoF)" patterns, as they are commonly known, are based upon key principles that have been recognized as supporting reuse.

\subsubsection{Open closed principle (OCP)}

First recorded by Meyer [10], OCP states that classes should be open to extension, but closed to modification. That is, it should be possible to extend the behavior of a class in response to new or changing requirements, but modification to the source code is not allowed. While these expectations may seem at odds, the key is abstraction. In $\mathrm{OO}$ a superclass can be created that is fixed, but can represent unbounded variation by subclassing.

\subsubsection{Once and only once (OAOO)}

While certainly not a new idea, Beck [12] put a name to the principle that any aspect of a software system - be it an algorithm, a set of constants, documentation, or logic - should exist in only one place. This isolates future changes, makes the system easier to comprehend and maintain, and through the low coupling and high cohesion that the principle instills, the reuse potential of these aspects increases. The encapsulation of state and behavior in objects, and the ability to inherit properties between classes allows for the rigorous application of these ideas in an object-oriented system, but is difficult to implement in structured techniques.

\subsubsection{Dependency inversion principle (DIP)}

The DIP states that high-level modules should not depend upon low-level modules. Both should depend upon abstractions. Which can be restated as: abstractions should not depend upon details, details should depend upon abstractions. Martin [13] introduced this idea as an extension to OCP with reference to the proliferation of dependencies that exist between highand low-level modules. For example, in a structured decomposition approach, the high-level procedures reference the lower level procedures, but changes often occur at the lowest levels. This infers that high-level modules or procedures that should be unaffected by such detailed modifications may be affected due to these dependencies. Again, consider the case where the camera characteristics change and even though perhaps only one routine needs to be rewritten, the calling module(s) need to me modified and recompiled as well. A preferable situation is to invert these dependencies such as is evident in the Liskov Substitution Principle.

\subsubsection{Liskov substitution principle (LSP)}

Liskov [9] expressed the principle of substitutivity of subclasses for their base classes as:

"If for each object $\mathrm{O}_{1}$ of type $\mathrm{S}$ there is an object $\mathrm{O}_{2}$ of type $\mathrm{T}$ such that for all programs $\mathrm{P}$ defined in terms of $T$, the behavior of $\mathrm{P}$ is unchanged when $\mathrm{O}_{1}$ is substituted for $\mathrm{O}_{2}$ then $\mathrm{S}$ is a subtype of T."

This principle has led to the concept of type inheritance and is the basis of polymorphism in objectoriented systems, where instances of derived classes can be substituted for each other provided they fulfill the obligations of a common superclass.

\subsubsection{Interface segregation principle (ISP)}

ISP states that "many client specific interfaces are better than one general purpose interface" [13]. This is, in essence, an extension of the idea of separating a module's interface from its implementation and therefore allowing them to vary independently. In cases where a class has many clients, designers should create specific interfaces for each client and multiply inherit them into the class. This approach ensures that any changes made to methods called by one client are not propagated to other clients with the payoff that those 
unaffected clients do not need recompilation or redeployment.

\subsection{Design patterns}

While the principles detailed above describe desirable aspects of object-oriented systems their systemic nature restricts their appropriate application during design to experts. Less advanced developers need more prescriptive, specific examples of the ideas expressed in the principles and this has led to the development of design patterns. Unfortunately research has shown that most designers of imaging systems are in this category [3].

To disseminate the ideas expressed in the principlesrobust, extensible, reusable software design - to the wider community Gamma et al. [14] developed a catalogue of design patterns. Each pattern addresses a common problem and then describes the core of the solution to that problem [15]. Many patterns languages, as they are termed, have since been created so most practitioners refer to Gamma's design patterns as the "Gang Of Four", or "GoF", patterns in recognition of the four authors.

There are 23 GoF patterns, too many to describe in detail here. Of interest to this work, however, is the appropriate application of these design patterns, usually in concert, to framework development in general, and imaging frameworks in particular. In subsequent sections two imaging frameworks will be examined for the presence of these patterns so those identified later will be described now.

\subsubsection{Abstract factory}

"Provide an interface for creating families of related or dependent objects without specifying their concrete classes.' [14]

The abstract factory pattern is applied in situations where it is desirable to control the creation of instances in a system such that clients of those instances are only aware of their interfaces, not their implementations, and families of related objects are used together and this constraint must be enforced. Rather than have domain objects create other instances a factory is defined that is responsible for instantiation of products thus ensuring that related objects are of the same family (when manipulating GIF images, only GIF codec elements and data structures are created, and not some GIF elements and a JPEG quantization table, for example).

\subsubsection{Singleton}

"Ensure a class only has one instance, and provide a global point of access to it."[14]
The Singleton pattern is often used in conjunction with the Abstract Factory, as it is often desirable to have only one instance of a factory per family, but the pattern is applicable beyond this example. In many situations it is necessary to allow only own instance of a class to exist and a simple way to achieve this is to make the class responsible for maintaining its only instance by defining a static, or class, method that returns the instance if it already exists, otherwise creates it.

\subsubsection{Observer}

"Define a one-to-many dependency between objects so that when one object changes state, all its dependents are notified and updated automatically.'”[14]

In general the GoF patterns encourage low coupling and high cohesion within a design, and the Observer pattern is a prime example. If an object is interested in changes in another object (a subject) it could call the subjects accessor methods (getSomething ()) periodically. This is undesirable, however, if many objects are similarly interested as it results in many couplings and the need for all those interested objects to know what information the subject maintains (its implementation). An alternative is to have the subject send updates to the interested objects, but ensuring that the subject equally does not know anything about the implementation of the interested observers. This is achieved by having observers implement an interface that the subject will use to call with updates, and having the subjects implement an interface that allows observers to subscribe (and unsubscribe) to those updates.

\subsubsection{Façade}

"Provide a unified interface to a set of interfaces in a subsystem. Facade defines a higher-level interface that makes the subsystem easier to use.'[14]

Another pattern intended to reduce coupling is the Façade. This is used to limit the communication and dependencies between subsystems by eliminating direct couplings between objects in different subsystems. This is achieved by defining a façade object that provides a single simple access point to the services of the subsystem and directs incoming requests to the appropriate objects within the subsystem to fulfill those requests. This shields the implementation of a subsystem from its clients thus promoting reuse, isolating internal objects from change, and generally reducing complexity. 


\subsubsection{Strategy}

"Define a family of algorithms, encapsulate each one, and make them interchangeable. Strategy lets the algorithm vary independently from clients that use it."[14]

A central feature of $\mathrm{OO}$ already mentioned is the encapsulation of data and behavior in a single entity, which can be restated as giving entities the means to manipulate and transform their own state. This gives rise to a problem, however, if there are many variants to the transforms of an object. Either the variants must be chosen with conditional logic (which is static and problematic to maintain and extend) or the object is subclassed with one variant per subclass (which is also static and possibly counter-intuitive; a list can be sorted in many ways, but it is always the same list, not a BubbleSortableList or a QuickSortableList, etc.). In such cases the Strategy pattern can be applied where the variant algorithms are encapsulated in their own classes and each implements the same interface so that clients are only abstractly coupled to the algorithm. This results in process-oriented classes which seems to counter the original premise of $\mathrm{OO}$, but better supports extension, maintenance and dynamic (runtime) change.

\section{Imaging frameworks}

Concrete examples of frameworks for imaging have been developed for the Java platform. Two of these, the Java Image I/O and 2D ${ }^{\mathrm{TM}}$ API's, will be investigated with reference to the patterns and principles of design. These are constituent packages of the Java Advanced Imaging (JAI) API that cannot be discussed in detail here given its considerable size and complexity-over 300 classes and interfaces are defined in the JAI API.

\subsection{Java image $I / O A P I$}

The Java Image I/O API is an example of a framework that provides a pluggable architecture for working with images stored in files and accessed across a network [16]. The API comprises six packages defining 46 classes and interfaces and supports extension through format-specific plugins created by subclassing core abstract classes, implementing defined interfaces and parameterizing subclasses with metadata. A partial class diagram of this API is presented in Fig. 1.

The notation used is from the Unified Modeling Language (UML) where classes are rendered as boxes with three partitions (for identity, attributes, and methods), interfaces are boxes with only two partitions (an interface does not have attributes), and relationships are indicated with lines. Four types of relationship can be indicated:

- generalization (inheritance) using an a solid line with an open triangle at the superclass end,

- dependency using a dashed directed line pointing to the entity depended upon,

- realization (implementation of an interface) using a dashed line with an open triangle at the interface end, and

- association using a solid line with, optionally, a diamond at one end indicating composition in a whole-part association.

\subsubsection{API description}

The central class in this model is the ImageIO class. This provides a set of static convenience methods for locating and calling ImageReaders and ImageWriters and creating ImageInputStreams and ImageOutputStreams.

Images are represented in the API by the BufferedImage class. This is a subclass of the abstract class java.awt.image and comprises a Color Model (an abstract class that encapsulates the methods for translating a pixel value to color components and an alpha component) and a Raster (an array of pixels). The Raster itself comprises a SampleModel, an abstract class that specifies the type of samples in the Raster and defines an interface for extracting these samples from an image.

The BufferedImage also inherits (termed "implements" in Java) the WriteableRenderedImage interface which is a subclass of the RenderedImage interface. It is this RenderedImage interface that is required by the Image Writer class, an abstract superclass responsible for image writing and encoding that implements the ImageTranscoder interface. Instances of ImageWriter subclasses (The API provides default ImageWriter subclasses for JPEG and PNG image formats) are instantiated by a service provider interface subclass of the Image WriterSpi abstract class.

Another service provider subclass (of the ImageReaderSpi abstract class) is responsible for instantiating instances of ImageReader subclasses. ImageReader is an abstract superclass for decoding and reading image files and, like ImageWriter, has default subclasses for JPEG and PNG, and also for GIF.

The final aspect of the Image I/O API shown in Fig. 1 is the EventListener interfaces (IIOWriteProgress Listener, IIOReadProgressListener, etc.). ImageReader and ImageWriter subclasses utilize a subscribe-publish protocol to update interested clients in the progress of image reading and writing.

\subsubsection{Evidence of patterns}

While the Image I/O API exhibits many examples of the OO principles described earlier, it makes use of 


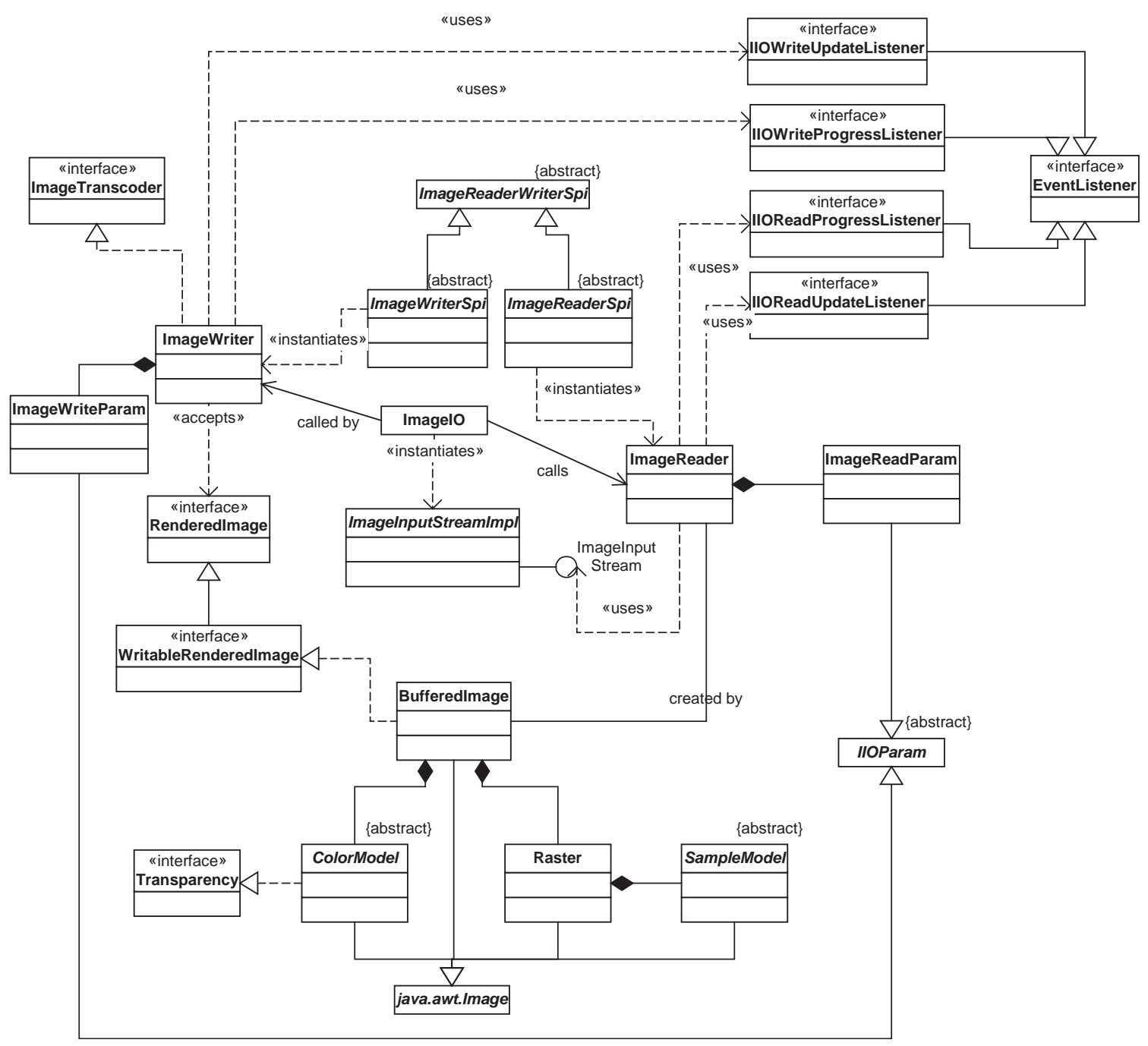

Fig. 1. Partial class diagram of Java Image I/O API.

relatively, and surprisingly, few of the GoF design patterns; at least in their common form. In the following subsections the evident patterns will be discussed.

4.1.2.1. Observer. The most obvious pattern evident in the Image I/O API is the Observer pattern. This is the GoF term for the publish-subscribe protocol described above with reference to the EventListener interfaces. Client objects interested in the state of ImageWriter instances can add themselves to the list of $I I O W$ riteProgressListeners (using the addIIOWriteProgress Listener() method). During image writing the ImageWriter will then call the imageProgress method of each listener notifying them of the progress (as a percentage) of the image being written. This obviates then need to modify ImageWriter subclasses to handle different listening classes if and when the need for such listeners arises. In fact, this is replace concrete (or implementation) coupling with abstract (interface) coupling and so supports low coupling, a key to reuse.
4.1.2.2. Façade. The ImageIO class provides a set of methods to clients of the ImageIO package. This is a form of façade since the methods provides clients a simple unified interface to the underlying package facilities. These methods include:

- getImageReaders(Object input) that returns all currently registered ImageReaders, that claim to decode that input,

- getImageReader(ImageWriter writer) that returns the corresponding ImageReader for that writer,

- and multiple read() and write() methods that can take URL, File or Stream parameters.

The primary benefit here is the reduction in external couplings to the package which reduces complexity and promotes subsystem independence.

4.1.2.3Singleton. All service provider interfaces (Image ReaderSpi, ImageWriterSpi, etc.) are registered with the 
IIORegistry (not shown in Fig. 1 for space reasons). Only one registry instance can exist, so the class is responsible for keeping track of its sole instance. This is achieved with the getDefaultInstance() class method that returns the instance if it exists else creates it.

4.1.2.4. Abstract factory. The service provider interface hierarchy resembles that of the abstract factory. For example, to create ImageReader instances the IIORegistry is searched for ImageReaderSpi instances and the appropriate instance is selected for the format type of the image to be read. This spi instance is then used to create the ImageReader instance for that image format. The ImageReaderSpi is an abstract class responsible for instantiating instances of ImageReader subclasses. This controls and localizes the creation of ImageReader instances ensuring that the correct reader is created.

\subsubsection{Framework extension}

The key characteristic of frameworks mentioned earlier is the ability to tailor the framework for a given application while leveraging the core services insulated within the abstractions. The design pattern constructs discussed above contribute to this facility, but the greater contribution in the Image I/O API seems to be in the application of the broader OO principles. That is to say that sound object-oriented design is more evident than specific design patterns.

The influence of the OCP is clear in the consistent use of interfaces and abstract classes that allow users to specialize classes such as ImageReader, ImageWriter, ColorModel, SampleModel and service providers to support various image formats. This reliance upon abstractions also supports the DIP. ImageWriter instances accept any image class that supports the RenderedImage interface rather than being dependent on concrete, or indeed more specialized abstract classes such as the BufferedImage class. This is also the case with regard to the listener classes. By conforming to standard interfaces any object can be updated with the progress of image reading and writing and the readers and writers have no knowledge of the implementation aspects of those clients obviating the need for future changes to support new interested observers (OCP at work again). The separate listener interfaces can also be considered as interface segregation as suggested by the ISP. Individual observers may be interested in reading progress, reading updates and reading warnings so can implement all three interfaces, rather than a single "readinglistener" interface, thus allowing the three aspects to vary independently if necessary.

Finally, the fact that most of the classes in the API are abstract classes, and therefore need to be subclassed and implemented by a framework user, the LSP is applied. Indeed, in almost all cases the associations and dependencies between classes in the package are at the abstract level therefore supporting the substitutivity of subclasses for their base classes.

\subsection{Java $2 D^{\mathrm{TM}} A P I$}

The Java $2 \mathrm{D}^{\mathrm{TM}}$ API enhances the imaging capabilities of the Abstract Windowing Toolkit enabling the development of advanced graphic libraries and image filters. It comprises six entire packages and parts of two others defining, in total, 127 classes and interfaces. The API supports three imaging models compared below in Table 1 [17].

Table 1

Features of Java 2D ${ }^{\mathrm{TM}}$ API supported imaging models (adapted from [17])

\begin{tabular}{|c|c|c|c|}
\hline & $\begin{array}{l}\text { Major interfaces/ } \\
\text { classes }\end{array}$ & Pros & Cons \\
\hline \multirow{2}{*}{$\begin{array}{l}\text { Push Model } \\
\text { (producer/consumer) }\end{array}$} & Image & Processing driven by image availability & Requires transfer of complete images \\
\hline & $\begin{array}{l}\text { ImageProducer } \\
\text { ImageConsumer } \\
\text { ImageObserver }\end{array}$ & Images processed incrementally & Complex programming interface \\
\hline \multirow[t]{2}{*}{$\begin{array}{l}\text { Immediate Mode } \\
\text { (image buffer model) }\end{array}$} & BufferedImage & Simple programming interface & $\begin{array}{l}\text { Requires memory allocation of } \\
\text { complete images }\end{array}$ \\
\hline & $\begin{array}{l}\text { Raster } \\
\text { BufferedImageOp } \\
\text { RasterOp }\end{array}$ & Commonly used model & Requires processing of complete images \\
\hline Pull Model (pipeline) & $\begin{array}{l}\text { RenderableImage } \\
\text { RenderableImageOp } \\
\text { (Java 2D API) } \\
\text { ImageConsumer } \\
\text { Image Observer } \\
\text { (Java Advanced } \\
\text { Imaging API) }\end{array}$ & $\begin{array}{l}\text { Stores/processes only required data } \\
\text { Allows lazy evaluation }\end{array}$ & $\begin{array}{l}\text { More complex programming interface } \\
\text { More complex implementation }\end{array}$ \\
\hline
\end{tabular}


Given the overall size of the API this discussion will be limited to the framework aspects related to the immediate mode-imaging model. A partial class diagram containing the classes and interfaces associated with this aspect is provided in Fig. 2 and those associated with jpeg compression (the sun.com.image. codec.jpeg package of the 2D API) are shown in Fig. 3.

\subsubsection{API description}

The BufferedImage class and its associated classes (ColorModel, Raster and SampleModel) from the Image I/O API are included in the 2D API. Additionally, the 2D API includes predefined subclasses for the ColorModel and SampleModel for different types of image formats (ComponentColor Model and ComponentSample Model for example). More interestingly, however, are the type hierarchies of the image operation classes (Raster $O p$ and BufferedImageOp). Many developers new to object-orientation find such seemingly processoriented classes in contradiction to the basic premise of the paradigm; the encapsulation of data and behavior into discrete entities. It is important to note, however, the intent of the "Fine-grained objects" framework pattern described earlier-break objects down into increasingly finer granularities until further decomposition results in objects with no relevance to the domain. In this case it is clear that image transforms are very relevant to the domain, and what's more, are likely to change in the future as new transforms are developed. In such cases it is important to encapsulate this potential variation. The API therefore defines several classes of image operations which implement both the Raster $O p$ and BufferedImageOp interfaces (and one class, BandCombine Op, that implements only the RasterOp interface).

The JPEG compression aspects are also included here to identify how different codecs can be defined within the framework. The central class is the JPEGCodec factory that creates instances of encoder and decoder classes that implement the JPEGEncoder and

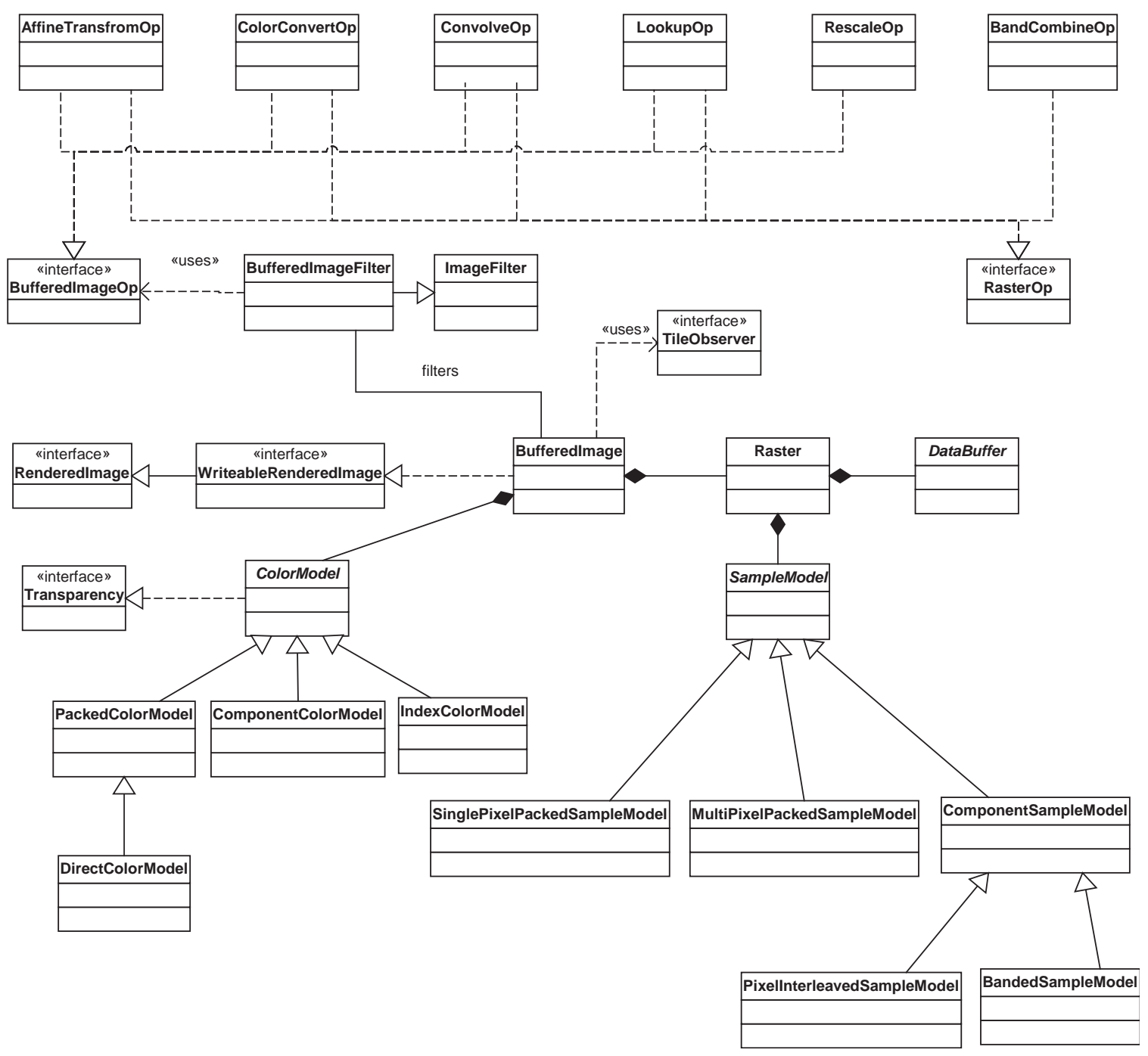

Fig. 2. Partial class diagram of Java $2 \mathrm{D}^{\mathrm{TM}}$ API (imaging classes and interfaces). 


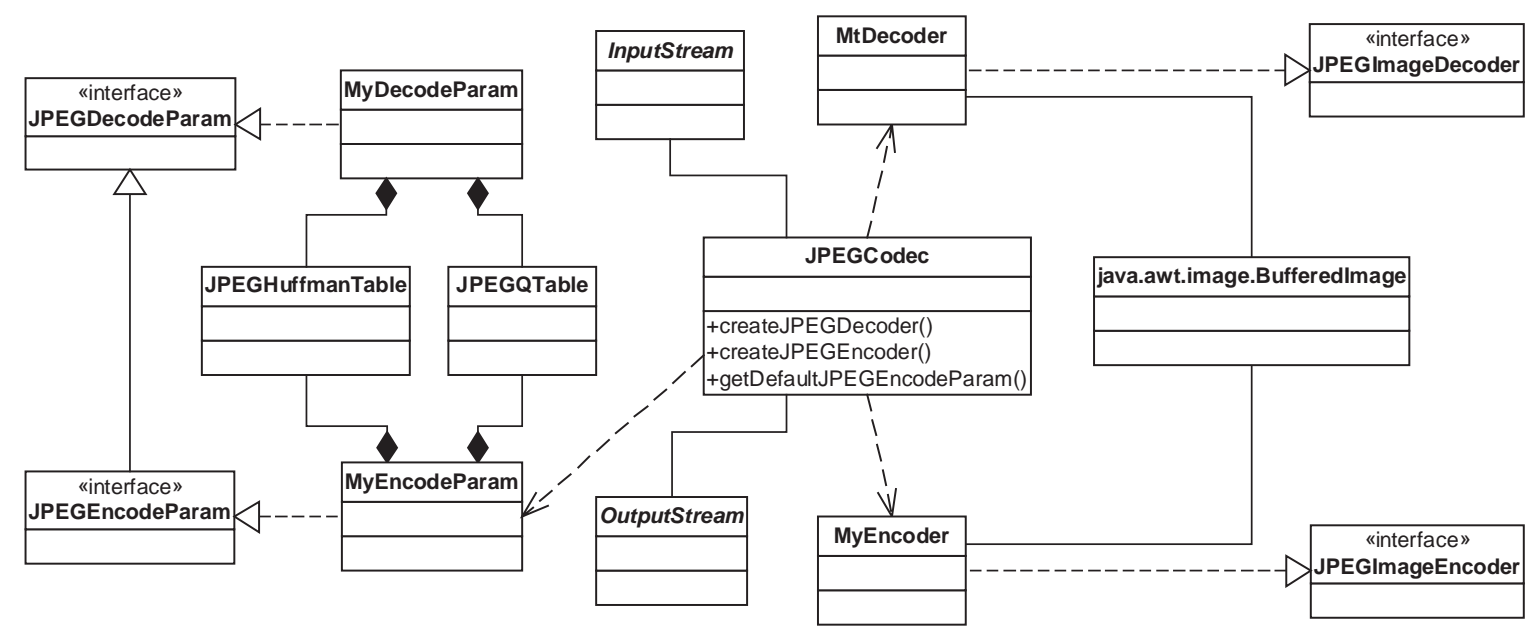

Fig. 3. Class diagram of com.sun.image.codec.jpeg package.

JPEGDecoder interfaces (indicated in Fig. 3 as $M y E n$ coder and MyDecoder) providing each with a parameter class that encapsulates the tables and options necessary to control coding(classes that implement the JPEGDecodeParam or JPEGEncodeParam interfaces) a stream (InputStream and OutputStream) and an image class (BufferedImage or Raster).

\subsubsection{Evidence of patterns}

4.2.2.1. Abstract factory. The Abstract Factory (or, more appropriately in this case, Factory) is the most obvious example of a pattern in the 2D API. The $J P E G C o d e c$ class is responsible for creating instances of encoders and decoders and with supplying these classes with the appropriate parameter, stream, and image classes. This approach ensures that the correct sets of classes are instantiated together (the correct parameter class for the coder class) and localizes the creation mechanism in case of future changes (OAOO principle).

4.2.2.2. Observer. The Observer pattern is also obvious in the API given the naming convention used. The TileObserver interface is an interface that must be implemented by any object interested in the state of tiles (modifiable or unmodifiable) in any object implementing the WriteableRenderedImage interface, such as BufferedImage objects. This obviates the need for image objects to "know" about the implementation of those objects interested in the images current state thus allowing for new types to be defined that wish to respond to changes in image objects without modification of the existing software (the new types just have to implement the TileObserver interface, and add themselves to the list of observers of the image object).

4.2.2.3. Strategy. The strategy pattern is apparent in Fig. 2 where image processing operations have been encapsulated in classes that implement common interfaces (Raster $O p$ and BufferedImageOp). Clients that might encapsulate such transforms (BufferedImage Filter, for example) now delegate the processing to these classes thus allowing the transforms to vary independently, and providing support for dynamic changes in processing (since the interfaces are inherited the derived classes are substitutable for one another-LSP).

\subsubsection{Framework extension}

As in the case of the Image I/O API, the 2D API defines an extensible framework and therefore reflects the object-oriented principles described earlier. In general the preponderance of interfaces and abstract classes within the API supports the OCP and LSP ideals of separating interface from implementation, and embedding common and non-variant aspects in the abstract levels.

In particular the ISP is evident in having the image operation classes implement two interfaces, one for each type of client that may be operated upon (BufferedImage or Raster) and therefore allowing the two interfaces to vary independently. To extend an application for more transforms the developer need only to implement one or both of these interfaces in the new operation class.

Extension to support additional codecs is not as mechanized, however. Examining the jpeg package it is evident that no generic (abstract) class of codec is defined nor any interfaces for the codec elements. Nevertheless, the general structure of future codecs is clear: define a factory "codec" class that instantiates an encoder and decoder and supplies them with any appropriate parameters, within their own class, along with the necessary InputStream, OutputStream, BufferedImage or Raster. It is also worth noting that in the Java Advanced Imaging API [18] (not discussed here because of its volume) a more generalized structure for codec extension is defined. 


\section{Conclusions}

In general software systems must cope with change or be rebuilt. Given that change is forced upon software systems almost continuously this exerts significant pressure upon the design. It is clearly undesirable to redevelop and redeploy a system whenever a new technology, standard, algorithm or device appears. This is exactly the situation in real-time imaging systems, however. As new image processing algorithms and standards are developed the software that support imaging systems must be modified and rebuilt. An alternative approach examined in this work leverages the widely documented advantages of object-oriented development. By applying some fundamental principles of good design a system can be constructed that is robust with respect to changing requirements, allows extension of functionality without modification to existing components and can be reused in any number of specific applications.

Two examples of such systems taken from the Java imaging APIs (Java Image I/O API and Java 2D ${ }^{\mathrm{TM}}$ API) have been described and the influence of the principles, and the more specific design patterns, is clear. The work also serves, therefore, as a demonstration of the practical application of design patterns, a compelling form of best practices, but it must be said that these are but examples of the fundamental principles in specific situations and are not the sole vehicle of reusable design.

\section{Acknowledgements}

I would like to acknowledge David Cloutier for his comments in personal communications on the application of object-oriented techniques for concurrent and real-time systems and to Dr. Laplante for his insightful review of this work.

\section{References}

[1] The Standish Group, The Chaos Report, 1995-2002, available at http://www.standishgroup.com.

[2] Pereira CE. Are object-oriented concepts useful to real-time systems development? Real-Time Systems, 2000;18(1):89-94.

[3] Laplante PA, Neill CJ. Software specification and design for imaging systems. Journal of Electronic Imaging, 2003;12(2):252-62.

[4] Laplante PA, Neill CJ. An overview of software specification techniques for real-time imaging. Proceedings of the real-time imaging conference. San Jose: SPIE; 2002. p. 55-64.

[5] Bollela G, Gosling J, Dibble P, Brosgul B, Furr S, Hardin D, Turnbull M. The real-time specification for Java. Reading: Addison-Wesley; 2000.

[6] Wedde HF. Critical issues in object-oriented real-time systems - a guided panel discussion. Real-Time Systems 2000;18(1):69-70.

[7] Bergmans L, Aksit M. Composing synchronization and real-time constraints. Journal of Parallel and Distributed Computing 1996;36(1):32-52.

[8] Binder RV. Testing objects: myth and reality. Object Magazine 1995;5(2):73-5.

[9] Liskov B. Data abstraction and hierarchy. SIGPLAN Notices 1988;23(5):17-34.

[10] Meyer B. Object-oriented software construction, 2nd ed. Engelwood Cliffs: Prentice-Hall; 2000.

[11] Lutz RR, Mikulski IC. Evolution of safety-critical requirements post-launch. In: Proceedings of the fifth IEEE international symposium on requirements engineering, Toronto, August 2001. p. 222-7.

[12] Beck K. Extreme programming explained: embrace change. New York: Addison-Wesley; 1999.

[13] Martin RC. The dependency inversion principle. C ++ Report, May 1996.

[14] Gamma E, Helm R, Johnson R, Vlissides J. Design patterns: elements of reusable object-oriented software. New York: Addison-Wesley; 1994.

[15] Alexander C, Ishakawa S, Silverstein M, Jacobson M, FiksdahlKing I, Angel S. A pattern language. New York: Oxford University Press; 1977.

[16] Race P, Rice D, Vera R. Java Image I/O API Guide, April 2001, available at http://java.sun.com/j2se/1.4/docs/guide/imageio/.

[17] Programmer's Guide to the Java 2D ${ }^{\mathrm{TM}}$ API: Enhanced Graphics and Imaging for Java, Sun Microsystems, Version 1.4, April 2001, available at http://java.sun.com/j2se/1.4/docs/guide/2d/index.html.

[18] Java Advanced Imaging API Specification, Sun Microsystems, Final Release 2, March 2001, available at http://java.sun.com/ products/java-media/jai/docs/index.html\#apidoc. 